\title{
Recombinant factor VIII in the management of hemophilia A: current use and future promise
}

This article was published in the following Dove Press journal:

Therapeutics and Clinical Risk Management

18 May 2009

Number of times this article has been viewed

\section{Jerry S Powell}

Division of Hematology and Oncology, University of California Davis Cancer

Center, Sacramento, CA, USA
Correspondence: Jerry S Powell Professor of Medicine, Hematology and Oncology, Division of Hematology and Oncology, Suite 3016, University of California Davis Cancer Center, 450I $X$ Street, Sacramento, CA 95817, USA Tel +19167348616

Fax + I 9167347946

Email jspowell@ucdavis.edu
Abstract: Hemophilia A is a rare inherited bleeding disorder due to mutation of the gene that encodes the coagulation protein factor VIII. Historically, prior to the availability of treatment with factor VIII preparations, most boys died from uncontrolled bleeding, either spontaneous bleeding or after injury, before reaching 20 years of age. One of the most impressive triumphs of modern medicine is that with current recombinant factor VIII replacement therapy, a boy born in the 21 st century with severe hemophilia A can anticipate a normal life expectancy with essentially no permanent complications from bleeding. For severe hemophilia A, current optimal treatment should have two goals: first, to provide sufficient factor VIII to prevent spontaneous bleeding, and second, to provide sufficient factor VIII to have normal coagulation function after any trauma. However, the replacement therapy requires tremendous resources for effective use, and remains extraordinarily expensive. Thus there are opportunities for further advances in therapy for hemophilia A. Two major concerns continue to trouble current optimal treatment approaches: some patients will develop neutralizing antibodies during the first 50 infusions of therapeutic factor VIII, and second, to administer therapeutic factor VIII every other day in young boys often requires placement of a central venous access device, and such use carries the life-threatening risks of infection and thrombosis. Because of the effectiveness of current therapy, any new developments in treatment will require significant concerns for safety, both immediate and in the long term. A number of research groups seek to prolong the biological efficacy of infused recombinant factor VIII. Currently, one such promising development is in the advanced stages of clinical trial. The goals will be to improve further the quality of life of an individual with severe hemophilia $\mathrm{A}$, and to reduce the burden of current treatment strategies on families and medical resources. Hopefully, the hemophilia community will continue to participate actively in the clinical trials needed to address these new challenges.

Keywords: factor VIII, hemophilia A, prophylaxis treatment, recombinant proteins

\section{Introduction}

Hemophilia A is a rare inherited bleeding disorder due to mutation of the gene that encodes the coagulation protein factor VIII. Severe hemophilia A is a condition almost exclusively found in males as the gene is located on the $\mathrm{X}$ chromosome; the incidence of hemophilia A is approximately 1 in 5000 male births. Approximately half of the sons of carrier females are affected, but more than $50 \%$ of the boys born with severe hemophilia A have no family history to suggest the need for carrier testing. ${ }^{1,2}$ Historically, prior to the availability of treatment with factor VIII preparations, most boys died from uncontrolled bleeding, either spontaneous bleeding or after injury, before reaching 20 years of age. ${ }^{3}$ Those who survived suffered from the complications 
of frequent bleeding, primarily severe and incapacitating damage involving their weight bearing joints. Intracranial hemorrhage occurred with an incidence of approximately one in 200 per year, often with severe permanent brain damage in the boys that survived the initial bleeding episode. ${ }^{4}$ One of the most impressive triumphs of modern medicine is that with current recombinant factor VIII replacement therapy, a boy born in the 21st century with severe hemophilia A can anticipate a normal life expectancy with essentially no permanent complications from bleeding. ${ }^{5}$ However, the replacement therapy requires tremendous resources for effective use, and remains extraordinarily expensive. ${ }^{6}$ Thus there are opportunities for further advances in therapy for hemophilia A.

\section{Historical developments in hemophilia A: up to $\mathbf{2 0 0 8}$}

In 1960 the average life expectancy for severe hemophilia A was less than 20 years, and the quality of life was generally devastating from joint bleeding complications or intracranial hemorrhage. ${ }^{7,8}$ The severity of hemophilia A depends primarily on the concentration of factor VIII activity in the blood, and is divided based on clinical phenotype into severe ( $<1 \%$ of normal circulating factor VIII activity), moderate ( $1 \%-4 \%)$, and mild ( $5 \%-25 \%)$. The work of Judith Graham Poole and others led to fractionation of blood, the serendipitous discovery of cryoprecipitate, and eventually to the use of plasma-derived preparations of factor VIII. Through improvements in the preparation of these plasmaderived products, and efforts to increase availability, there was sufficient supply of factor VIII preparations so that most boys with severe hemophilia A were treated when spontaneous or traumatic bleeding episodes occurred, a style of therapy termed "on-demand" treatment, as factor VIII was administered to treat bleeding after it had already started, rather than therapy to prevent bleeding at all. With vigorous medical support primarily through hemophilia treatment centers and home treatment programs, and with plasmaderived factor VIII products widely available, the death rate for hemophilia A had improved dramatically by the late 1970s. In a recent prospective study, it was found that patients with severe hemophilia who attended clinic at hemophilia treatment centers in the US experienced $40 \%$ less mortality than patients who did not visit hemophilia treatment centers, reflecting the need for comprehensive expertise and resources in treating this disorder. ${ }^{9}$ Such successful comprehensive treatment centers are being developed around the world. ${ }^{10}$ Joint damage was much less in most boys, but still severe, resulting in marked compromise in their quality of life. ${ }^{11}$ However, spontaneous intracranial hemorrhage remained a significant morbidity even in aggressive on demand treatment programs, with neonatal hemorrhage a particular management problem even in known female carriers. ${ }^{12,13}$

Then in the early 1980s, the epidemic of HIV devastated the hemophilia community, and by 1985 nearly $75 \%$ of the severe hemophilia A patients in the US had acquired HIV. Also, many of the moderate and mild hemophilia A patients, who used factor VIII only occasionally, were also infected. The ironic tragedy was that the source of the HIV infection was the plasma-derived factor VIII products that had promised to be life-saving. The virus had entered the blood supply, was carried into the plasma preparations, and proved to be highly contagious for the patients with severe hemophilia A when they received plasma preparations for bleeding episodes as often as twice a week. More than half of these infected individuals died from HIV by 1995, when effective treatment for HIV infection first became widely available. ${ }^{11}$

Amidst the confusion and anger during the early years of the HIV epidemic, the response of a significant number of individuals with hemophilia A was to stop taking any plasma-derived products completely, preferring to return to the clinical situation of 1960 , with the consequences of severe bleeding poorly treated.

As the community dealt with the horrors of HIV, a second epidemic gradually became apparent. Over $95 \%$ of severe hemophilia A patients had also acquired hepatitis $\mathrm{C}$ infection through plasma-derived preparations of factor VIII. ${ }^{14}$ There were several reasons that realization of the severity of the epidemic of hepatitis $\mathrm{C}$ developed slowly in the hemophilia community. Most medical attention was concentrated on dealing with the HIV epidemic, a much more rapid and devastating illness. Hepatitis $\mathrm{C}$ virus was not identified until 1989. Infection with hepatitis C rarely causes acute illness, is eliminated by the immune system approximately $20 \%$ of the time, often remains completely asymptomatic, and, when symptomatic, becomes a chronic medical problem, often minimally symptomatic for years until manifesting primarily as liver damage worsening over years, with cirrhosis and liver failure apparent 10 to 20 years later, or liver cancer apparent 20 to 30 years after infection. Thus, for patients with severe hemophilia A infected by plasma-derived preparations in the late 1970s and early 1980s, these complications became widely apparent only in the late 1990s.

Fortunately, for individuals with hemophilia A, four independent developments have occurred over the past 
20 years that have improved their clinical situation again. These developments support optimistic predictions of normal life expectancy for boys born with hemophilia A in the $21 \mathrm{st}$ century, and for effective treatments for older individuals who have acquired the twin infections of HIV and hepatitis $\mathrm{C}$. First, effective treatments have been developed for HIV infection. Many of the patients with hemophilia A infected initially in the early 1980s have had their HIV infection converted to a chronic manageable condition that, while not cured, allows them to live a relatively normal life again. Second, treatments that provide cure for significant numbers of individuals infected with hepatitis $\mathrm{C}$ have been developed and new promising treatments are developing relatively rapidly. Current treatment for hepatitis $\mathrm{C}$ is arduous, but cure rates of up to $40 \%$ have been reported in hemophilia A patients.

Two other developments have been critical for hemophilia A treatment. Preparations of the plasma-derived products have been improved: steps have been added to test donor populations that provide plasma for purification of factor VIII, steps have been added to inactivate any viruses present, and additional steps of factor VIII purification have been included. The success of these efforts is demonstrated by the fact that no transmission of HIV, hepatitis C, or other virus has been documented to be associated with any of these modern plasma-derived factor VIII preparations since 1990. This safety record for these products ranks among the best for any pharmaceutical product. ${ }^{15-18}$

The fourth major development for hemophilia A over the past 20 years has been the provision of recombinant factor VIII, widely available shortly after completion of the clinical trials in 1994. Several preparations of recombinant factor VIII are currently available for patients with hemophilia A. The World Federation of Hemophilia provides a summary listing of all currently available factor VIII products and their major characteristics; the latest update is edition 8 (April 2008). ${ }^{19}$ The development of recombinant factor VIII has been a major step forward in the treatment of hemophilia $\mathrm{A} .{ }^{20}$ There is not consensus regarding whether any one of the currently available recombinant factor VIII products has significant clinical advantages over the others in any particular clinical setting. Each product has a strong safety record, has been available for a number of years, and is licensed in many countries. Each of the products has its proponents; randomized clinical trials comparing these products are unlikely. Potential improvements for recombinant factor VIII include advances that would allow prevention of spontaneous bleeding with fewer intravenous infusions. Besides the obvious convenience to the patient of fewer infusions required to prevent spontaneous bleeding, there are medical advantages as well. The current rate of septic arthritis in males with hemophilia is approximately 83 per 100,00 person-years compared with only 2 to 6 per 100,000 person-years in non-hemophilia. ${ }^{21}$ Central venous access device use and infection with hepatitis $\mathrm{C}$ or HIV were not risk factors for septic arthritis in this study; presumably the risk factor was related to multiple intravenous infusions. Advances in the effective half life of recombinant factor VIII would be expected to reduce the incidence of septic arthritis in hemophilia.

The history of hemophilia A treatment over the past 40 years has been tumultuous: with two major epidemics in the 1980 s, but ending with the availability of recombinant factor VIII, and current expectations of essentially normal life expectancy for this previously devastating genetic disease. Most of the hemophilia community probably views this past 40 years as a story of survival, and with relief, rather than a story of triumph. Perhaps with more years to provide greater historical perspective, the tragedies of epidemics will fade and the scientific triumph of recombinant factor VIII will become the dominant story, along with the change in life expectancy for severe hemophilia A boys from less than 20 years to normal life expectancy, achieved in less than 30 years of medical progress.

\section{Current treatment for hemophilia A}

For severe hemophilia A, current optimal treatment should have two goals: first, to provide sufficient factor VIII to prevent spontaneous bleeding, and second, to provide sufficient factor VIII to have normal coagulation function after any trauma. At the present time, optimal treatment so defined requires much medical support and family support, primarily due to the relatively short half-life of infused factor VIII, approximately 10 hours. Until more effective treatment alternatives are available, optimal treatment as defined here both requires tremendous effort and is extraordinarily expensive, and thus often requires any of a number of compromises that lead to adequate, but sub-optimal, therapy.

Factor VIII requires intravenous infusion, and is bound rapidly to its carrier molecule von Willebrand protein. Without effective interaction with von Willebrand protein, the infused factor VIII disappears rapidly in minutes due to degradation by plasma proteases, as occurs in the subtype of von Willebrand Disease, Type N (for Normandy, where it was first described), in which the protein domain of von Willebrand protein that interacts with factor VIII 
is rendered ineffective by mutations. With a half-life of 10 hours, intravenous infusion of factor VIII is required approximately every other day to maintain factor VIII concentrations high enough to provide adequate support for coagulation to prevent spontaneous bleeding. The therapeutic goal is to achieve a clinical situation that is roughly similar to individuals with moderate hemophilia $\mathrm{A}$ in whom the concentration of factor VIII activity is more than $2 \%$ by standard clotting assays. These boys with moderate hemophilia A rarely experience spontaneous bleeding, although traumatic bleeding remains problematic. Thus, the assumption initially was that maintenance of factor VIII activity greater than $2 \%$ would be necessary to prevent any spontaneous bleeding episodes. However, numerous studies over the past 20 years have demonstrated that while clotting assays are pretty accurate in predicting the clinical phenotype of individuals with hemophilia A, there is significant variation that is much more difficult to predict, again confirming that coagulation is indeed a complex problem. Many proteins need to interact to provide effective coagulation and protection against spontaneous bleeding, and many boys do not need intravenous infusion of factor VIII as often as every other day to be protected from spontaneous bleeding episodes. In this population, the clotting assays do not correlate as closely as needed with protection from spontaneous bleeding. Proper understanding of the clinical situation and some means to identify which boys will need every other day infusions are critical because currently such a rigorous infusion schedule often requires the placement of a central venous access device with attendant risks for medical complications. ${ }^{22}$ These complications could be avoided by predicting which boys do not require such frequent infusions.

In severe hemophilia A, spontaneous bleeding will occur in some boys during the third day after infusion of factor VIII. After several months, with many such "third days" with factor VIII concentrations less than $2 \%$, most boys will experience spontaneous bleeding. However, some boys essentially never bleed spontaneously despite factor VIII levels of less than $2 \%$ for many days, allowing these boys to receive infusions only once each week to protect them from spontaneous bleeding. ${ }^{23,24}$ These considerations have led Canadian investigators to advocate a graduated response to treatment for severe hemophilia $\mathrm{A}$, in which recombinant factor VIII is infused once each week until the boy experiences unacceptable spontaneous bleeding, at which time infusions are escalated to twice each week. Then in these boys, further spontaneous bleeding would lead to further escalation of infusions to 3 times per week, and then to every other day if necessary. Their initial encouraging experience with this approach has been published, and indeed, such an approach avoids the use of central venous access devices in many boys, avoiding the medical risks of such catheters. ${ }^{25,26}$

The major concern in this graduated escalation approach for infusions of factor VIII to prevent spontaneous bleeding is that many boys will experience spontaneous bleeding, raising the critical questions of how much spontaneous bleeding can occur in any single joint and how much joint damage can be sustained before the joint suffers irreversible damage. ${ }^{27}$ In one study delayed starting of prophylaxis until after several joint bleeds led to progression of arthropathy and deterioration of joint function over time. ${ }^{28}$ The subsidiary clinical question is then how much damage can the joint sustain before it significantly compromises the quality of life of the individual with severe hemophilia. One of the major advances now happening in the hemophilia community in the 21 st century is the gradual realization that these more subtle questions affecting hemophilia outcomes will be answerable only through properly conducted prospective clinical trials that compare different therapeutic approaches. ${ }^{29}$ One of the first major prospective clinical trials addressing these questions was recently published. ${ }^{30}$ This trial compared prophylactic infusions in young children to prevent any spontaneous bleeding versus on-demand treatment infusions with recombinant factor VIII. The regular prophylactic infusion schedule led to reduced spontaneous bleeding and much less joint damage. In addition, joints suffering only one or two spontaneous bleeding episodes had detectable joint damage several years later, suggesting that the joint damage may indeed be permanent, and perhaps progressive, once the process has been initiated by bleeding.

The above discussion considered only spontaneous joint bleeding, but similar results are typical for spontaneous intracranial hemorrhage in severe hemophilia patients: those on every other day full prophylaxis, with the target goal being factor VIII activity nadirs greater than $1 \%$, very rarely experience intrancranial bleeding, while those patients using on-demand therapy continue to experience spontaneous intracranial bleeding and neurological sequelae. ${ }^{31,32}$ Whether gradually escalating schedules of factor VIII infusions risk intracranial bleeding is not clear yet.

Numerous questions remain to be addressed in future clinical studies. During the development of recombinant factor VIII, randomized prospective trials were not needed to demonstrate efficacy, primarily because the differences between no treatment and factor VIII infusion were dramatic, and there is an excellent correlation between clinical phenotype 
for bleeding risk and the coagulation laboratory test of factor VIII activity. Now, however, the differences in outcomes between different approaches of therapy with factor VIII preparations are much more subtle, and the answers to support evidence based clinical decisions will depend on randomized prospective clinical trials. ${ }^{33}$ In this context, it is hoped that more and more hemophilia A patients and their treating doctors will demand that the hemophilia community answer these questions through randomized studies, rather than through competing expert opinions or through political decisions that allocate limited resources. For example, is the quality of life of adult hemophilia A patients better using the graduated escalating frequency of infusions to prevent most spontaneous bleeding or using the regular infusion schedule of every other day to prevent essentially all spontaneous bleeding $?^{34,35}$ Do the medical complications outweigh the benefits? ${ }^{36,37}$ What additional therapies are beneficial when treating the complications of severe hemophilia A? ${ }^{38-41}$ Comparison data from clinical trials will be important to guide appropriate decisions for where and how society should allocate its health care resources. ${ }^{42}$

Even with recombinant factor VIII there is significant variability in the pharmacokinetic profiles between individuals with severe hemophilia A, as significant as previously experienced with plasma-derived factor VIII preparations. Anecdotal experience indicates also that some patients will have improved half life for infused therapeutic factor VIII with one product over another, but this observation has not been rigorously tested in randomized clinical trials. Therefore it is difficult to predict which products will benefit one patient more than another product, or even whether switching products will provide benefit in any individual patient. ${ }^{43}$ Variables that influence the pharmacokinetic profile in individual patients need additional study, ${ }^{44}$ but appear to include variation in surface receptors that affect factor VIII binding and metabolism. ${ }^{45}$

Prevention of spontaneous bleeding episodes is an important goal in hemophilia care. Another important consideration will be how to treat optimally for minor traumatic bleeding. Spontaneous bleeding is rare in boys with factor VIII activity of more than $2 \%$. However, in active boys, of course, minor traumatic bleeding often occurs in the presence of higher concentrations of factor VIII. Do these traumatic bleeds cause the same risks for permanent joint damage as spontaneous bleeding? Are there better tests, either genetic or biochemical laboratory tests, that may define populations of boys at greater risk for damage from minor traumatic bleeds? And, are there programs for clinical management that lead to better outcomes for these growing boys ${ }^{46}$ Since coagulation is the result of complex interactions involving over a dozen proteins, and each of those proteins has biological differences, the clinical situation in hemophilia $\mathrm{A}$ is quite variable, and bleeding frequencies do not reflect solely the activity obtained from factor VIII laboratory testing. ${ }^{47}$ There is a significant need for new coagulation tests that allow prediction of which boys would benefit from the different approaches to treatment of their severe hemophilia A.

\section{Complications of current treatment of hemophilia A}

Two major concerns continue to trouble current optimal treatment approaches for severe hemophilia A. First, some patients will develop inhibitors (antibodies that neutralize the activity of the FVIII molecule) during the first 50 infusions of therapeutic factor VIII. Second, to administer therapeutic factor VIII every other day in young boys often requires placement of a central venous access device, and such use carries the risks of infection and thrombosis, either of which complication may be life-threatening. Much effort is directed currently at understanding which boys are at risk for inhibitors, and which are at risk for complications of central venous access devices.

The incidence of neutralizing inhibitor formation varies depending on the population studied, but generally the incidence of inhibitor formation is $15 \%$ to $20 \% .{ }^{48}$ Since most individuals with severe hemophilia A are missing large portions, if not all, of the factor VIII protein, the immune systems in these individuals would be expected to perceive the epitopes of the missing factor VIII molecule as "foreign" and trigger an immune response against those foreign epitopes. ${ }^{49,50}$ From this perspective, then, it is surprising that therapeutic infusions with factor VIII do not trigger formation of neutralizing inhibitors in most of the recipients with severe hemophilia A. Perhaps the factor VIII molecule lacks immunogenicity in many individuals due to sequence similarity with other proteins such as factor $\mathrm{V}$, but the reasons for only a small proportion of patients developing neutralizing antibodies are not well understood. With continued infusion of factor VIII to induce a form of immune tolerance, some of these inhibitors disappear, the half-life of infused factor VIII becomes again normal, and these individuals can be treated again as other patients with severe hemophilia A. With addition of immune-modulatory therapy along with continued infusions of factor VIII, a few more of these neutralizing inhibitors disappear. The remaining individuals, approximately $5 \%$ to $8 \%$ of severe hemophilia patients, are treated with NovoSeven ${ }^{\circledR}$ 
(Novo Norkisk), a preparation of activated factor VII, and/or FEIBA (Factor VIII Inhibitor Bypassing Activity), a plasmaderived preparation of coagulation factors that has been partially activated during the preparation process. ${ }^{51,52}$ It is encouraging that the use of these products to treat hemophilia patients with inhibitors is guided by results from randomized clinical trials. ${ }^{29}$ The half-life of each of these preparations is much shorter than factor VIII, leaving these inhibitor patients with a higher frequency of spontaneous bleeding. Treatment of trauma and coagulation support for surgery is essentially as effective with these alternative preparations in inhibitor patients as factor VIII preparations in patients without inhibitors. ${ }^{53}$

A major question in modern therapy for hemophilia revolves around how to predict which boys with severe hemophilia A will develop inhibitors. Younger siblings of boys who have developed high titers of neutralizing inhibitors, are at markedly increased risk of inhibitor development, suggesting that individual immune systems account for some of the variation in inhibitor development. Genetic analyses of the individual gene defects for hemophilia A are beginning to help provide additional understanding. ${ }^{54}$ As would be expected, absence of large portions of the gene, or mutations that lead to absence of large portions of the protein correlate well with higher risks of inhibitor development, presumably because the immune system senses the infused therapeutic factor VIII as a foreign protein, since most T-cell and B-cell epitopes of factor VIII are absent in these boys with large deletions of protein. Other mutations in factor VIII may create novel epitopes in the endogenously produced FVIII, so that the individual immune systems sense these regions of the infused therapeutic FVIII as antigenic to varying degrees. Differences in epitope profile may also correlate with the success of immune tolerance induction after the development of a high titer inhibitor to factor VIII. Such differences in B-cell epitopes, for example, in the FVIII C2 and A2 domains may be associated with failure to achieve immune tolerance induction with current approaches. Inclusion of the carrier molecule for FVIII, von Willebrand protein, may somehow modulate the immune recognition of these B-cell epitopes. ${ }^{55,56}$ Continuing research will expand the data bases of genetic differences underlying hemophilia A, and how these genetic differences correlate with risks for inhibitor development, and with the response to immune tolerance induction. ${ }^{57}$

In addition to genetic variables in mutations of the factor VIII molecule and in the immune system, environmental variables may be important also. Interpretations of the clinical data in this area of inhibitor formation are controversial and more studies should help clarify the significant risk factors for inhibitor formation. Currently, some studies suggest that young children exposed to factor VIII in the setting of activated immune reactions, as in infections and/or during and after surgery, may have higher risk of developing inhibitors. Other studies support the concern that recombinant factor VIII is associated with higher risk of inhibitor formation. ${ }^{58,59}$ Some of these studies support the hypothesis for a mechanism of inhibitor formation due to the more highly purified factor VIII protein in the recombinant preparations. ${ }^{60}$ Other interpretations suggest that the association of the natural carrier protein, von Willebrand protein, with the infused therapeutic factor VIII provides some undefined mechanism that decreases the formation of inhibitors. ${ }^{61,62}$ Inhibitor development is a poorly understood phenomenon, and there are few randomized clinical trials. A list of putative environmental factors that may influence inhibitor development includes: 1 ) is the age of first exposure critical, 2) is higher dosing more important as an independent risk factor at young ages, 3 ) is the setting of first use of factor VIII infusion critical to triggering the immune response of inhibitor development, that is, in the setting of activation of the immune system after surgery, infection or trauma critical for increased risk of inhibitor development, 4) is the presence of the carrier protein von Willebrand factor in the infused factor VIII preparation critical for protection against inhibitor development. ${ }^{63}$ Then, complicating the interpretation of anecdotal data from small numbers of patients who develop inhibitors is whether the answers regarding environmental contributions are different depending on the individual genetic mutations that cause hemophilia A.

In such situations when facing many questions about what therapy is associated with better outcomes, other areas of medicine have used prospective randomized clinical trials to compare the therapeutic options. Several hurdles to this approach complicate use of randomized trials in the hemophilia community. Perhaps most important among these barriers is the emotional reluctance of both families and hemophilia physicians to "subjecting" young boys to randomization of the choice of factor VIII preparations. This reluctance is understandable in view of the past history of two life-threatening epidemics transmitted to patients with hemophilia through plasma-derived factor VIII preparations. However, as there has been no transmission of any significant virus through plasma-derived products since 1990, the reassurance of this extensive experience is beginning to balance the fear of transmission of a new virus. Conversely, there are some hemophilia physicians who advocate using only 
von Willebrand protein containing factor VIII preparations in view of the evidence suggesting that the rate of inhibitor development is lower with these preparations than with recombinant factor VIII. ${ }^{59}$ Currently, this decision requires using plasma-derived factor VIII preparations. Until the hemophilia community achieves some consensus on how to address this issue, randomized trials are unlikely to start. Fortunately, research in new factor VIII preparations may allow a third alternative to avoid this dilemma. If the fear is the potential danger of plasma-derived products, and the advantage is using von Willebrand protein containing preparations, then the third alternative is to prepare recombinant von Willebrand protein to infuse along with recombinant factor VIII. Several pharmaceutical companies are pursuing research aimed at providing such a preparation.

The second major concern troubling current treatment of hemophilia A involves the complications from use of central venous access devices to administer factor VIII every other day. The incidence of these complications, primarily infections and thromboses, varies considerably, depending on the population involved, on the experience and expertise of the hemophilia treatment center, and on the efforts of the families involved in home infusion for each individual child with severe hemophilia A. Some apparent conclusions emerge from most studies of the complications of use of such catheters. First, the complication rate is highest in children under 2 years of age, and yet, these are the individuals in whom venous access every other day is most problematic. Second, experience and training of the primary care provider in the home setting is critical to avoiding complications of catheter use. Third, there appear to be some boys in whom risks of complications are high for reasons not easily identified; some boys repeatedly acquire infections or thromboses. There may be unknown genetic risk factors involved in immune response genes or coagulation that predispose certain individuals to complications of catheter use. Further studies may identify such factors. Currently, concern for the risks of central catheters has led to a number of treatment options that may compromise the optimal treatment of infusion every other day.

\section{Are all currently available recombinant factor VIII products interchangeable?}

This question is a difficult one to answer, and the clear evidence-based response is that it cannot be answered fully until there are prospective randomized clinical trials comparing treatment using product A with treatment using product B. Such clinical trials are unlikely to happen, in part because such trials would be extraordinarily expensive. After that disclaimer, there are some differences considered important by some clinicians. Recombinant factor VIII products are divided subjectively into 4 groups. The first three groups are defined by amounts of human plasmaderived protein contained in the final factor VIII product, and are generally listed as first-, second-, and third-generation recombinant factor VIII products. The first generation products used human albumin in the media to grow the cell lines that produced the recombinant factor VIII. Since factor VIII is a relatively unstable molecule albumin was used to help stabilize the factor VIII in the final product. The second generation products used less albumin at each stage of development and production. ${ }^{64}$ The third generation products used essentially no human plasma-derived proteins in the development or production stages. ${ }^{65}$ All three generations of recombinant factor VIII are based on the full length gene with very minor differences in sequences that are not significant. Two different mammalian cell lines are used to produce these three generations of recombinant factor VIII, leading to measurable differences in the glycosylation of the factor VIII protein that is infused. Since factor VIII is a highly glycosylated molecule there were intensive studies to detect clinically significant differences between the molecules. ${ }^{66}$ None was identified.

For the three generations of full length recombinant factor VIII there are many studies in patients with hemophilia A, but none that directly compares one generation with another product or generation. Given that lack of direct comparison, however, one can attempt to compare the results of the various clinical studies, and, given the lack of direct comparison data, it appears that there is no detectable difference in effective half-life, in efficacy to treat bleeds, either spontaneous or traumatic, in efficacy during surgery, in inhibitor formation in children who have never been previously treated with factor VIII products, in inhibitor formation in individuals who have been previously treated with other factor VIII products, or in complications during infusions. After over a decade of experience with these three generations of recombinant factor VIII products in hemophilia A, the choice of which product to use is driven by ease of administration, insurance company reimbursement decisions, perceived concerns regarding the theoretical potential of viral transmission, ${ }^{67}$ and social issues. Most clinicians regard these products as essentially equivalent, and allow the patient 
and/or his family or his insurance to decide which of these products to infuse.

The fourth group of recombinant factor VIII is defined based on its lacking the B-domain of the factor VIII molecule. ${ }^{68}$ The $\mathrm{B}$ domain is the central portion of the full length protein that is removed during synthesis in normal liver cells, and constitutes nearly one third of the molecular weight of the factor VIII molecule. Interestingly, the B domain is relatively highly conserved through evolution, and yet no physiological function has been identified for the B domain. Presumably, it represents an example of the lack of efficiency of evolution, in that evolutionary pressures apply only to protein sequences that carry survival disadvantages, and even large protein domains can survive through evolution, as long as they do not convey disadvantageous characteristics.

Interestingly, the B-domainless recombinant FVIII has not enjoyed the commercial success of the full length recombinant factor VIII products. Perhaps part of the lack of acceptance in the hemophilia community was reflected in questions regarding efficacy. Initially Gruppo et al raised questions regarding its clinical efficacy, despite uncontrolled cohort clinical studies essentially equivalent to similar studies using the full length plasma-derived or full length recombinant factor VIII. ${ }^{69}$ In a meta-analysis of 13 such clinical studies they reported finding reduced hemostatic efficacy during prophylaxis and shortened half-life of B domain deleted recombinant factor VIII compared with full length factor VIII. ${ }^{70}$ As they stated, clearly such metaanalyses lack the power of studies that directly compare the products. Such direct comparison studies were performed using plasma-derived factor VIII (Hemophil M) and separately using full length recombinant factor VIII (Advate ${ }^{\circledR}$; Baxter Healthcare). The direct pharmacokinetic comparisons showed that the factor VIII products are bioequivalent to each other. ${ }^{68,71}$

\section{Current treatment of hemophilia} adults with recombinant factor VIII - how much is optimally safe?

Almost two generations of patients with severe hemophilia A have experienced the benefits of the widely available factor VIII preparations. After the crises of two blood product transmitted viral infections, the hemophilia community now has many senior citizens with severe hemophilia A. This marked increase in adults with severe hemophilia A is a blessing from the medical progress of the last century, but now the community is facing for the first time large numbers of hemophilia patients with concomitant medical problems that may interact with hemophilia in strange and unpredictable ways. ${ }^{72}$ Establishing the data to support evidence based clinical decisions for these patients will be a challenge. ${ }^{73}$ There is some suggestion that the risk of spontaneous intracranial hemorrhage in individuals with severe hemophilia A, highest in the neonatal period, may increase again in older patients due to other risk factors such as age, hypertension, and arteriosclerosis. As another example, consider that myocardial infarction results primarily from acute thrombosis of coronary arteries. Thus, does severe hemophilia A reduce the risk of developing atherosclerosis, and/or of suffering from myocardial infarction? Tuinenburg et al recently published a review that concluded hemophilia was associated with a relatively lower risk of cardiovascular disease, but that the incidence was increasing as the population with hemophilia ages.$^{74}$ However, in populations with normal coagulation, elevated factor VIII activity is associated with increased risk of cardiovascular events, including myocardial infarction..$^{75}$ Since current treatment for hemophilia A uses episodic infusion of factor VIII, is there a risk associated with infusions that achieve peak factor VIII activity concentrations of greater than $100 \%$ ? Do adults have different risks from these prophylactic infusions compared to children without co-morbidities? A different set of questions revolve around the use of recombinant factor VIII versus plasma-derived factor VIII preparations in adults. Elevated von Willebrand protein is also an independent risk factor for cardiovascular and thrombotic disease..$^{75}$ Is there a risk associated with the use of von Willebrand protein containing plasma-derived factor VIII preparations in adults with concomitant cardiovascular disease? ${ }^{76}$ Will a different set of clinical guidelines for infusion therapy need to be developed for older adults with severe hemophilia A? Such questions will be answered only through clinical investigation.

\section{Current promising developments in therapy with recombinant factor VIII}

Current therapy of hemophilia A has achieved essentially normal life expectancy for boys born today with severe hemophilia A. Thus, any new developments in treatment will require significant concerns for safety, both immediate and in the long term. These safety concerns will justifiably slow the development of novel treatments for severe hemophilia A. ${ }^{77}$ In active clinical research are two approaches 
that illustrate alternative strategies for improved hemophilia treatment.

A number of research groups seek to prolong the biological efficacy of the infused recombinant factor VIII. One such promising development is in advanced stages of clinical trial. ${ }^{78,89}$ In this approach, the recombinant factor VIII molecule is non-covalently attached to a pegylated liposome molecule prior to infusion. In two phase I clinical trials this preparation was shown to be safe for at least one infusion in patients with severe hemophilia A, and also to prolong the interval free of spontaneous bleeding after prophylactic infusion. ${ }^{79-82}$ Using this new formulation of recombinant factor VIII, there is a pivotal non-inferiority trial currently underway with enrollment expected to be completed in the third quarter of 2009. Each subject will be followed for one year, with randomization to compare clinical efficacy using standard recombinant factor VIII infused three times each week, with efficacy using the pegylated liposomal factor VIII infused once a week. This clinical trial should provide results in 2010. Other approaches not yet in clinical trial include genetic modifications of factor VIII to extend the half-life after infusion, to use gene therapy approaches to express normal factor VIII, and genetic modifications to extend the half life of factor VII to allow effective coagulation in the absence of normal factor VIII. ${ }^{77,83-85}$

Particularly intriguing may be the approach to modify the balance of coagulation so that spontaneous bleeding occurs much less frequently. In vivo, coagulation requires the reaction complex of factor IX and its cofactor factor VIII to activate factor X rapidly, in part because tissue factor pathway inhibitor limits the rate of the reaction of factor VIIa to activate factor X. One of the mechanisms by which NovoSeven treats bleeding in severe hemophilia A patients is believed to be that the limiting activity of tissue factor pathway inhibitor on the reaction of factor VIIa to activate factor X directly is overwhelmed by large concentrations of factor VIIa, thus essentially bypassing the need for any activity of the coagulation complex of factor IX/factor VIII. Using in vitro models of coagulation, it has been demonstrated that inhibition of the limiting activity of tissue factor pathway inhibitor allows coagulation to proceed as rapidly as addition of large concentrations of activated factor VIIa, as rapidly as severe hemophilia A plasma treated with factor VIII, and as rapidly as normal plasma or whole blood. ${ }^{86}$ An oral compound that inhibited tissue factor pathway inhibitor might allow an individual with severe hemophilia A to avoid any spontaneous bleeding and thus, to avoid the need for intravenous infusions of factor VIII entirely except for bleeding associated with trauma or surgery. Clinical trials will be needed to evaluate the efficacy and safety of such inhibitors.

\section{Thoughts on current controversies in optimal treatment of severe hemophilia A with recombinant factor VIII}

Within the hemophilia community there are many different opinions regarding optimal treatment for hemophilia A, reflecting the tumultuous past 3 decades of exciting new developments in treatment and the experiences of two devastating viral epidemics transmitted through promising new treatment products. The community needs more data to provide evidence to support treatment guidelines. ${ }^{87,88}$ Three controversies will be addressed here, partly in hopes of encouraging the community to consider participation in prospective clinical trials.

Spontaneous intracranial bleeding is a frightening complication of severe hemophilia A. It occurs essentially only when the factor VIII activity is less than $2 \%$, and it occurs primarily in children under the age of 4 , in whom the use of central venous access devices is associated with higher risk of serious infectious or thrombotic complications. Thus, some clinicians eschew the use of vascular access in these children in fear of the complications, while other clinicians embrace their use in order to prevent intracranial hemorrhage and any neurological damage. Multicenter prospective trials are needed to define the balancing point where benefits outweigh the risks, and which procedures are associated with optimal outcomes.

In the hemophilia community over the past few years, current treatment schedules with recombinant factor VIII, with the proven efficacy to prevent bleeding, and with the tremendous safety record, have led to a shift in the paradigm for treatment of severe hemophilia A. Previous studies have focused on preventing joint damage. The new paradigm is to prevent any spontaneous bleeding at all. This shift in thinking is analogous to earlier shifts in medicine with other disorders, such as treating hypertension rather than simply trying to prevent strokes, or such as treating blood sugar in diabetes rather than simply trying to prevent kidney damage. The defect in hemophilia A is a lack of factor VIII coagulation activity, and the goal of treatment should be replacement of factor VIII activity and prevention of any spontaneous bleeding. This new shift in considering how to treat severe hemophilia A is reflected in the US FDA's recent decision to grant an indication for use in prophylaxis to one of the recombinant factor VIII products currently available. 
This decision was based in part on a prospective randomized clinical trial that compared two treatment schedules using recombinant factor VIII for severe hemophilia A. ${ }^{30}$ This clinical trial compared prophylaxis treatment with on demand treatment in children, and clearly demonstrated in this young population the superiority of prophylaxis treatment with recombinant factor VIII. Other trials will need to address among other considerations, questions of appropriate dosing of factor VIII, whether plasma-derived factor VIII or other recombinant factor VIII products convey similar efficacy, and whether similar beneficial outcomes occur in adults with severe hemophilia A.

The third area for consideration reflects that for the past two decades the hemophilia community has pushed diligently for factor VIII that was safer, with safety defined as higher purity factor VIII protein with no associated human plasmaderived products. Now, the question is being refocused to: how safe is this definition of safety? ${ }^{1,2}$ No plasma-derived factor VIII preparation has transmitted any documented viral illness since 1990. The safety concern now revolves around fear of transmission of prions, or perhaps fear of "the next" as yet unidentified "virus", sometimes loosely named "TNV." Are these concerns still rational, in view of the two decades long safety record for all plasma-derived factor VIII preparations and the documented absence of known transmission of prion disease by any factor VIII preparation ${ }^{67}$ If the alternative risks were only theoretical or even perhaps only financial, then the concerns might be rational. However, in view of the accumulating evidence that high purity factor VIII products lacking von Willebrand protein and recombinant factor VIII may be associated with increased risk of inhibitor formation, the new question is which risk is greater for the hemophilia community? Thoughtful clinicians disagree on these issues, based on their personal experiences and in the absence of data supported conclusions based on prospective comparative clinical trials. Some clinicians use only recombinant factor VIII, so that children never exposed to blood products remain never exposed, because of fear of prions or TNV. Other clinicians use only plasma-derived von Willebrand protein containing factor VIII preparations because they are convinced that inhibitor development is significantly reduced, and they apparently view the concern of viral transmission as much less significant for their patients.

Will a way forward be found? Given the tremendous progress in hemophilia over the past 40 years, it is likely that the hemophilia community will indeed find a new development. Perhaps prospective clinical trials will be started to address the question directly. Perhaps recombinant von Willebrand protein will be developed and its addition to recombinant factor VIII will be tested in prospective randomized clinical trials. Perhaps the pegylated liposome factor VIII product currently in phase 3 clinical trial will prove further useful as a carrier molecule for factor VIII, and the risk of inhibitor formation will be as low as the heralded low risk for plasma-derived von Willebrand protein containing factor VIII products. Hopefully, the hemophilia community will participate actively in the clinical trials needed to address these questions. Of the many possibilities it is certain only that the future will be interesting.

\section{Summary}

The hemophilia community has achieved a remarkable goal for treatment of severe hemophilia A: normal life expectancy for a previously life threatening disease. Now, the challenges will be to improve further the quality of the life of an individual with severe hemophilia $A$, and to reduce the burden of current treatment strategies on families and medical resources. Promising new recombinant factor VIII products are in advanced stages of clinical trials, and studies are underway to address how to optimally use current resources to improve quality of life for individuals with severe hemophilia A.

\section{Disclosures}

The author declares no conflicts of interest.

\section{References}

1. Aledort LM. History of haemophilia. Haemophilia. 2007;13 Suppl 5:1-2.

2. Mannucci PM. Back to the future: a recent history of haemophilia treatment. Haemophilia. 2008;14 Suppl 3:10-18.

3. Larsson SA, Wiechel B. Deaths in Swedish hemophiliacs, 1957-1980. Acta Med Scand. 1983;214(3):199-206.

4. Ljung RC. Intracranial haemorrhage in haemophilia A and B. Br J Haematol. 2008;140(4):378-384.

5. Darby SC, Kan SW, Spooner RJ, et al. Mortality rates, life expectancy, and causes of death in people with hemophilia A or B in the United Kingdom who were not infected with HIV. Blood. 2007;110(3):815-825.

6. De Moerloose P, Urbancik W, Van Den Berg HM, Richards M. A survey of adherence to haemophilia therapy in six European countries: results and recommendations. Haemophilia. 2008;14(5):931-938.

7. Ikkalat E, Helske G, Myllyla H, et al. Changes in the life expectancy of patients with severe haemophilia A in Finland in 1930-79. Br J Haematol, 1982;52:7-12.

8. Triemstra M, Rosendaal FR, Smit C, Van der Ploeg HM, Briët E. Mortality in patients with hemophilia. Changes in a Dutch population from 1986 to 1992 and 1973 to 1986. Ann Intern Med. 1995;123(11):823-827.

9. Soucie JM, Nuss R, Evatt B, et al. Mortality among males with hemophilia: relations with source of medical care. The Hemophilia Surveillance System Project Investigators. Blood. 2000;96(2):437-442.

10. Poon MC, Luke KH. Haemophilia care in China: achievements of a decade of World Federation of Hemophilia treatment centre twinning activities. Haemophilia. 2008;14(5):879-888.

11. Plug I, Van Der Bom JG, Peters M, et al. Mortality and causes of death in patients with hemophilia, 1992-2001: a prospective cohort study. J Thromb Haemost. 2006;4(3):510-516. 
12. Stieltjes N, Calvez T, Demiguel V, et al; French ICH Study Group. Intracranial haemorrhages in French haemophilia patients (1991-2001): clinical presentation, management and prognosis factors for death. Haemophilia. 2005;11(5):452-458.

13. Mishra P, Naithani R, Dolai T, et al. Intracranial haemorrhage in patients with congenital haemostatic defects. Haemophilia. 2008;14(5):952-955.

14. Goedert JJ, Chen BE, Preiss L, Aledort LM, Rosenberg PS. Reconstruction of the hepatitis $\mathrm{C}$ virus epidemic in the US hemophilia population, 1940-1990. Am J Epidemiol. 2007;165(12):1443-1453.

15. Berntorp E, Archey W, Auerswald G, et al. A systematic overview of the first pasteurised VWF/FVIII medicinal product, Haemate P/ Humate -P: history and clinical performance. Eur J Haematol Suppl. 2008; (70):3-35.

16. Blanchette VS, Shapiro AD, Liesner RJ, et al; rAHF-PFM Clinical Study Group. Plasma and albumin-free recombinant factor VIII: pharmacokinetics, efficacy and safety in previously treated pediatric patients. J Thromb Haemost. 2008;6(8):1319-1326.

17. Mäkipernaa A, von Bonsdorff L. Fifteen years of population based experience in Finland with a plasma-derived monoclonal purified factor VIII concentrate. Haemophilia. 2008;14(3):612-614.

18. Mauser-Bunschoten EP, Posthouwer D, Fischer K, van den Berg HM Safety and efficacy of a plasma-derived monoclonal purified factor VIII concentrate during 10 years of follow-up. Haemophilia. 2007;13(6):697-700.

19. Brooker M. Registry of clotting factor concentrates, 8th ed, 2008; World Federation of Hemophilia. Available at: www.wfh.org.

20. Négrier C, Shapiro A, Berntorp E, et al. Surgical evaluation of a recombinant factor VIII prepared using a plasma/albumin-free method: efficacy and safety of Advate in previously treated patients. Thromb Haemost. 2008;100(2):217-223

21. Ashrani AA, Key NS, Soucie JM, Duffy N, Forsyth A, Geraghty S; Universal Data Collection Project Investigators. Septic arthritis in males with haemophilia. Haemophilia. 2008;14(3):494-503.

22. Neunert CE, Miller KL, Journeycake JM, Buchanan GR. Implantable central venous access device procedures in haemophilia patients without an inhibitor: systematic review of the literature and institutional experience. Haemophilia. 2008;14(2):260-270.

23. Jayandharan GR, Srivastava A. The phenotypic heterogeneity of severe hemophilia. Semin Thromb Hemost. 2008;34(1):128-141.

24. Shetty S, Ghosh K. Reduced clinical severity in a mutationally wellcharacterized cohort of severe hemophilia with associated thrombophilia. Am J Clin Pathol. 2008;130(1):84-87.

25. Biss TT, Chan AK, Blanchette VS, Iwenofu LN, McLimont M, Carcao MD; Association of Hemophilia Clinic Directors of Canada (AHCDC); Canadian Association of Nurses in Hemophilia Care (CANHC). The use of prophylaxis in 2663 children and adults with haemophilia: results of the 2006 Canadian national haemophilia prophylaxis survey. Haemophilia. 2008;14(5):923-930.

26. Risebrough N, Oh P, Blanchette V, Curtin J, Hitzler J, Feldman BM. Costutility analysis of Canadian tailored prophylaxis, primary prophylaxis and on-demand therapy in young children with severe haemophilia A. Haemophilia. 2008;14(4):743-752.

27. Manco-Johnson M. Comparing prophylaxis with episodic treatment in haemophilia A: implications for clinical practice. Haemophilia. 2007; 13 Suppl 2:4-9.

28. Löfqvist T, Nilsson IM, Berntorp E, Pettersson H. Haemophilia prophylaxis in young patients - a long-term follow-up. J Intern Med. 1997;241(5):395-400.

29. Mannucci PM. Need for randomized trials in hemophilia. J Thromb Haemost. 2006;4(3):501-502.

30. Manco-Johnson MJ, Abshire TC, Shapiro AD, et al. Prophylaxis versus episodic treatment to prevent joint disease in boys with severe hemophilia. N Engl J Med. 2007;357(6):535-544.

31. Ljung R, Chambost H, Stain AM, DiMichele D. Haemophilia in the first years of life. Haemophilia. 2008;14 Suppl 3:188-195.

32. Traivaree C, Blanchette V, Armstrong D, Floros G, Stain AM, Carcao MD. Intracranial bleeding in haemophilia beyond the neonatal period - the role of CT imaging in suspected intracranial bleeding. Haemophilia. 2007;13(5):552-559.
33. Collins PW, Blanchette VS, Fischer K, et al; On behalf of the rAHF-PFM study group. Break-through bleeding in relation to predicted factor VIII levels in patients receiving prophylactic treatment for severe haemophilia A. J Thromb Haemost. 2009;7(3):413-420.

34. Gringeri A, Von Mackensen S. Quality of life in haemophilia. Haemophilia. 2008;14 Suppl 3:19-25.

35. Tagliaferri A, Franchini M, Coppola A, et al. Effects of secondary prophylaxis started in adolescent and adult haemophiliacs. Haemophilia. 2008;14(5):945-951.

36. Schobess R, Kurnik K, Friedrichs F, et al. Effects of primary and secondary prophylaxis on the clinical expression of joint damage in children with severe haemophilia A. Results of a multicenter non-concurrent cohort study. Thromb Haemost. 2008;99(1):71-76.

37. Stobart K, Iorio A, Wu JK. Clotting factor concentrates given to prevent bleeding and bleeding-related complications in people with hemophilia A or B. Cochrane Database Syst Rev. 2006, Issue 2. Art. No: CD003429. DOI:10.1002/14651858.CD003429.pub3.

38. Jansen NW, Roosendaal G, Lafeber FP. Understanding haemophilic arthropathy: an exploration of current open issues. Br J Haematol. 2008; 143(5):632-640.

39. Lobet S, Pendeville E, Dalzell R, et al. The role of physiotherapy after total knee arthroplasty in patients with haemophilia. Haemophilia. 2008;14(5):989-998.

40. Rodriguez-Merchan EC. Ankle surgery in haemophilia with special emphasis on arthroscopic debridement. Haemophilia. 2008;14(5):913-919.

41. Tatsunami S, Mimaya J, Shirahata A, et al. Current status of Japanese HIV-infected patients with coagulation disorders: coinfection with both HIV and HCV. Int J Hematol. 2008;88(3):304-310.

42. Rentz A, Flood E, Altisent C, et al; Members of the HAEMO-QoL-A Steering Committee. Cross-cultural development and psychometric evaluation of a patient-reported health-related quality of life questionnaire for adults with haemophilia. Haemophilia. 2008;14(5):1023-1034.

43. Shapiro AD, Korth-Bradley J, Poon MC. Use of pharmacokinetics in the coagulation factor treatment of patients with haemophilia. Haemophilia. 2005;11(6):571-582.

44. Butenas S, Parhami-Seren B, Gissel MT, Gomperts ED, Fass DN, Mann KG. Potency and mass of factor VIII in FVIII products. Haemophilia. 2009;15(1):63-72.

45. Lenting PJ, Christophe OD, Guéguen P. The disappearing act of factor VIII. Haemophilia. 2008 Sep 1. [Epub ahead of print].

46. Fischer K, Valentino L, Ljung R, Blanchette V. Prophylaxis for severe haemophilia: clinical challenges in the absence as well as in the presence of inhibitors. Haemophilia. 2008;14 Suppl 3:196-201.

47. Lewis SJ, Stephens E, Florou G, et al. Measurement of global haemostasis in severe haemophilia A following factor VIII infusion. Br J Haematol. 2007;138(6):775-782.

48. DiMichele D. Immune tolerance therapy for factor VIII inhibitors: moving from empiricism to an evidence-based approach. J Thromb Haemost. 2007;5 Suppl 1:143-150.

49. Oldenburg J, Pavlova A. Genetic risk factors for inhibitors to factors VIII and IX. Haemophilia. 2006;12 Suppl 6:15-22.

50. Boekhorst J, Lari GR, D'Oiron R, et al. Factor VIII genotype and inhibitor development in patients with haemophilia A: highest risk in patients with splice site mutations. Haemophilia. 2008;14(4):729-735.

51. Berntorp E. Differential response to bypassing agents complicates treatment in patients with haemophilia and inhibitors. Haemophilia. 2009; 15(1):3-10.

52. Jiménez-Yuste V, Alvarez MT, Martín-Salces M, et al. Prophylaxis in 10 patients with severe haemophilia A and inhibitor: different approaches for different clinical situations. Haemophilia. 2009;15(1):203-209.

53. Teitel JM, Carcao M, Lillicrap D, et al. Orthopaedic surgery in haemophilia patients with inhibitors: a practical guide to haemostatic, surgical and rehabilitative care. Haemophilia. 2009;15(1):227-239.

54. Margaglione M, Castaman G, Morfini M, et al; AICE-Genetics Study Group. The Italian AICE-Genetics hemophilia A database: results and correlation with clinical phenotype. Haematologica. 2008;93(5): $722-728$. 
55. Greninger DA, Saint-Remy JM, Jacquemin M, Benhida A, DiMichele DM. The use of factor VIII/von Willebrand factor concentrate for immune tolerance induction in haemophilia A patients with high-titre inhibitors: association of clinical outcome with inhibitor epitope profile. Haemophilia. 2008;14(2):295-302.

56. Gringeri A, Musso R, Mazzucconi MG, et al; RITS-FITNHES Study Group. Immune tolerance induction with a high purity von Willebrand factor/VIII complex concentrate in haemophilia A patients with inhibitors at high risk of a poor response. Haemophilia. 2007;13(4):373-379.

57. Green PM, Bagnall RD, Waseem NH, Giannelli F. Haemophilia A mutations in the UK: results of screening one-third of the population. Br J Haematol. 2008;143(1):115-128.

58. Calvez T, Laurian Y, Goudemand J. Inhibitor incidence with recombinant vs plasma-derived FVIII in previously untreated patients with severe hemophilia A: homogeneous results from four published observational studies. J Thromb Haemost. 2008;6:390-392.

59. Gouw SC, van der Bom JG, Marijke van den Berg H. Treatment-related risk factors of inhibitor development in previously untreated patients with hemophilia A: the CANAL cohort study. Blood. 2007;109(11): 4648-4654.

60. Lacroix-Desmazes S, Navarrete AM, André S, Bayry J, Kaveri SV, Dasgupta S. Dynamics of factor VIII interactions determine its immunologic fate in hemophilia A. Blood. 2008;112(2):240-249.

61. Kreuz W. The role of VWF for the success of immune tolerance induction. Thromb Res. 2008;122 Suppl 2:S7-S12.

62. Federici AB, Mannucci PM, Marco P. Von Willebrand factor in highpurity factor VIII complex concentrates: chaperone protein or key to therapies? A meeting report. Haemophilia. 2008;14(1):133-139.

63. ter Avest PC, Fischer K, Mancuso ME, et al; CANAL Study Group. Risk stratification for inhibitor development at first treatment for severe hemophilia A: a tool for clinical practice. J Thromb Haemost. 2008;6(12):2048-2054.

64. Musso R, Santagostino E, Faradji A, et al; KOGENATE Bayer European PMS Study Group. Safety and efficacy of sucrose-formulated full-length recombinant factor VIII: experience in the standard clinical setting. Thromb Haemost. 2008;99(1):52-58.

65. Shapiro AD. Anti-hemophilic factor (recombinant), plasma/albumin-free method (octocog-alpha; ADVATE) in the management of hemophilia A. Vasc Health Risk Manag. 2007;3(5):555-565.

66. Singleton E, Smith J, Kavanagh M, Nolan B, White B. Low risk of inhibitor formation in haemophilia patients after a change in treatment from Chinese hamster ovary cell-produced to baby hamster kidney cell-produced recombinant factor VIII. Thromb Haemost. 2007;98(6): 1188-1192.

67. Wilson K, Ricketts MN. A third episode of transfusion-derived vCJD. Lancet. 2006;368(9552):2037-2039.

68. Kessler CM, Gill JC, White GC 2nd, et al. B-domain deleted recombinant factor VIII preparations are bioequivalent to a monoclonal antibody purified plasma-derived factor VIII concentrate: a randomized, threeway crossover study. Haemophilia. 2005;11(2):84-91.

69. Gruppo RA, Brown D, Wilkes MM, Navickis RJ. Comparative effectiveness of full-length and B-domain deleted factor VIII for prophylaxis a meta-analysis. Haemophilia. 2003;9(3):251-260.

70. Gruppo RA, Brown D, Wilkes MM, Navickis RJ. Increased breakthrough bleeding during prophylaxis with B-domain deleted factor VIIIa robust meta-analytic finding. Haemophilia. 2004;10(5):449-451.
71. Di Paola J, Smith MP, Klamroth R, Mannucci PM, Kollmer C, Feingold J, Kessler C, Pollmann H, Morfini M, Udata C, Rothschild C, Hermans C, Janco R. ReFacto and Advate: a single-dose, randomized, two-period crossover pharmacokinetics study in subjects with haemophilia A. Haemophilia. 2007;13(2):124-130.

72. Franchini M, Tagliaferri A, Mannucci PM. The management of hemophilia in elderly patients. Clin Interv Aging. 2007;2(3):361-368.

73. Dolan G, Hermans C, Klamroth R, Madhok R, Schutgens RE, Spengler U. Challenges and controversies in haemophilia care in adulthood. Haemophilia. 2009;15 Suppl 1:20-27.

74. Tuinenburg A, Mauser-Bunschoten EP, Verhaar MC, Biesma DH, Schutgens RE. Cardiovascular disease in patients with haemophilia. J Thromb Haemost. 2008 Oct 18. [Epub ahead of print].

75. Kulkarni R, Soucie JM, Evatt BL; Hemophilia Surveillance System Project Investigators. Prevalence and risk factors for heart disease among males with hemophilia. Am J Hematol. 2005;79(1):36-42.

76. Walsh CE; Adult Prophylaxis Study Group. Prophylaxis for adults with severe haemophilia: a compelling need for evidence-based guidelines. Haemophilia. 2008;14(2):385-386.

77. Lillicrap D. Extending half-life in coagulation factors: where do we stand? Thromb Res. 2008;122 Suppl 4:S2-S8.

78. Powell, JS. Liposomal approach towards the development of a longeracting factor VIII. Haemophilia 2007, 13 Suppl 2:23-28.

79. Powell JS, Nugent DJ, Harrison JA, Soni A, Luk A, Stass H, Gorina E. Safety and pharmacokinetics of a recombinant factor VIII with pegylated liposomes in severe hemophilia A. J Thromb Haemost. 2008; 6(2):277-283.

80. Martinowitz U, Lalezari S, Luboshitz J, Lubetsky A, Spira J. Infusion rates of recombinant FVIII-FS with PEGylated liposomes in haemophilia A. Haemophilia. 2008;14(5):1122-1124.

81. Spira J, Plyushch OP, Andreeva TA, Andreev Y. Prolonged bleedingfree period following prophylactic infusion of recombinant factor VIII reconstituted with pegylated liposomes. Blood. 2006;108(12): 3668-3673.

82. Spira J, Plyushch OP, Andreeva TA, Khametova RN. Evaluation of liposomal dose in recombinant factor VIII reconstituted with pegylated liposomes for the treatment of patients with severe haemophilia A. Thromb Haemost. 2008;100(3):429-434.

83. Kasuda S, Kubo A, Sakurai Y, et al. Establishment of embryonic stem cells secreting human factor VIII for cell-based treatment of hemophilia A. J Thromb Haemost. 2008;6(8):1352-1359.

84. Murphy SL, High KA. Gene therapy for haemophilia. Br J Haematol. 2008;140(5):479-487.

85. Ohmori T, Ishiwata A, Kashiwakura Y, et al. Phenotypic correction of hemophilia A by ectopic expression of activated factor VII in platelets. Mol Ther. 2008;16(8):1359-1365.

86. Prasad S, Lillicrap D, Labelle A, et al. Efficacy and safety of a newclass hemostatic drug candidate, AV513, in dogs with hemophilia A. Blood. 2008;111(2):672-679.

87. Oldenburg J, Dolan G, Lemm G. Haemophilia care then, now and in the future. Haemophilia. 2009;15 Suppl 1:2-7.

88. Pipe SW, Valentino LA. Optimizing outcomes for patients with severe haemophilia A. Haemophilia. 2007;13 Suppl 4:1-16.

89. Powell J. The next generation of anti-haemophilia factor, factor VIII. Long-lasting protection from spontaneous bleeding, are we there yet? Thromb Haemost. 2008;100(3):365-366.
Therapeutics and Clinical Risk Management

\section{Publish your work in this journal}

Therapeutics and Clinical Risk Management is an international, peerreviewed journal of clinical therapeutics and risk management, focusing on concise rapid reporting of clinical studies in all therapeutic areas, outcomes, safety, and programs for the effective, safe, and sustained use of medicines. This journal is indexed on PubMed Central, CAS,

\section{Dovepress}

EMBase, Scopus and the Elsevier Bibliographic databases. The manuscript management system is completely online and includes a very quick and fair peer-review system, which is all easy to use. Visit $\mathrm{http}: / /$ www.dovepress.com/testimonials.php to read real quotes from published authors. 